\title{
Elevation of erythrocyte sedimentation rate and C-reactive protein levels reflects renal interstitial inflammation in drug-induced acute tubulointerstitial nephritis
}

Xi-zi Zheng ${ }^{1}$, Yang-hui Gu ${ }^{1,2,3}$, Tao Su' ${ }^{1}$ Xu-jie Zhou ${ }^{1,2}$, Jun-wen Huang ${ }^{1,2}$, Ping-ping Sun ${ }^{1,2}$, Yan Jia ${ }^{1,2}$, Da-min Xu', Su-xia Wang ${ }^{1,2,4}$, Gang Liu ${ }^{1,2}$ and Li Yang ${ }^{1,2^{*}}$

\begin{abstract}
Background: A renal biopsy is needed to define active inflammatory infiltration and guide therapeutic management in drug-induced acute tubulointerstitial nephritis (D-ATIN). However, factors such as various contraindications, refusal of informed consent and limited technical support may stop the biopsy process. It is thus of great importance to explore approaches that could deduce probable pathologic changes.

Methods: A total of 81 biopsy-proven D-ATIN patients were enrolled from a prospective cohort of ATIN patients at Peking University First Hospital. The systemic inflammation score (SIS) was developed based on the CRP and ESR levels at biopsy, and patients were divided into high-SIS, median-SIS, and low-SIS groups. The demographic data, clinicopathologic features, and renal outcomes were compared.

Results: The SIS was positively correlated with inflammatory cell infiltration and was inversely correlated with interstitial fibrosis. The number of interstitial inflammatory cells increased significantly with increasing SISS. The proportions of neutrophils and plasma cells were the highest in the high-SIS group compared with the other two groups. Prednisone (30-40 mg/day) was prescribed in all patients. The high-SIS group tended to have more favorable renal restoration than the other two groups. By 12 months postbiopsy, a decreased eGFR $(<60 \mathrm{~mL} / \mathrm{min} /$ $1.73 \mathrm{~m}^{2}$ ) was observed in $66.7 \%$ of medium-SIS patients, $32.4 \%$ of high-SIS patients, and $30.4 \%$ of low-SIS patients.

Conclusion: The SIS was positively correlated with active tubulointerstitial inflammation and therefore could help to aid therapeutic decisions in D-ATIN.
\end{abstract}

Keywords: $\mathrm{C}$ reactive protein, Erythrocyte sedimentation rate, Interstitial inflammation, Drug-induced acute tubulointerstitial nephritis

\footnotetext{
* Correspondence: li.yang@bjmu.edu.cn

${ }^{1}$ Renal Division, Peking University First Hospital, Peking University Institute of Nephrology; Key Laboratory of Renal Disease, Ministry of Health of China; Key Laboratory of Chronic Kidney Disease Prevention and Treatment (Peking University), Ministry of Education, Beijing 100034, People's Republic of China ${ }^{2}$ Renal Pathology Center, Peking University Institute of Nephrology, Beijing 100034, People's Republic of China

Full list of author information is available at the end of the article
}

(c) The Author(s). 2020 Open Access This article is licensed under a Creative Commons Attribution 4.0 International License, which permits use, sharing, adaptation, distribution and reproduction in any medium or format, as long as you give appropriate credit to the original author(s) and the source, provide a link to the Creative Commons licence, and indicate if changes were made. The images or other third party material in this article are included in the article's Creative Commons licence, unless indicated otherwise in a credit line to the material. If material is not included in the article's Creative Commons licence and your intended use is not permitted by statutory regulation or exceeds the permitted use, you will need to obtain permission directly from the copyright holder. To view a copy of this licence, visit http://creativecommons.org/licenses/by/4.0/ The Creative Commons Public Domain Dedication waiver (http://creativecommons.org/publicdomain/zero/1.0/) applies to the data made available in this article, unless otherwise stated in a credit line to the data. 


\section{Background}

Acute tubulointerstitial nephritis (ATIN) is a common renal lesion histopathologically characterized by inflammation and edema of the renal interstitium. It is responsible for $15-27 \%$ of acute kidney injury (AKI) [1-5], with drugs being the most common cause [6-10]. Unlike ischemic or toxic AKI, which usually induces acute tubular injury and results in an abrupt decline in renal function, patients with drug-induced ATIN (D-ATIN) sometimes have insidious renal dysfunction and are therefore more likely experience delayed recognition. A renal biopsy is needed to make a definitive diagnosis of D-ATIN and reveals the activity and severity of interstitial inflammation that usually directs immunosuppressive treatment [11-13]. However, factors such as various contraindications, refusal of informed consent and limited technical support may stop the process of renal biopsy. It is thus of great importance for those patients clinically suspected of having D-ATIN, in whom renal biopsy cannot be conducted, to explore approaches that could deduce probable pathologic changes and indicate the severity of interstitial inflammatory cell infiltration, which could therefore help make therapeutic decisions.

The erythrocyte sedimentation rate (ESR) and Creactive protein (CRP) are traditional inflammatory markers that have been used to help assess the activity of inflammation in various diseases, such as systemic lupus erythematosus [14-17], rheumatoid arthritis [18, 19], and vasculitis [20]. However, there is still a lack of knowledge about the relevance of these systemic inflammatory parameters to renal inflammation. The current study was performed in a prospective cohort of D-ATIN patients. The clinical-pathological features and renal recovery of patients with different CRP and ESR levels were compared. Associations between systemic inflammation and renal tubulointerstitial inflammation as well as long-term renal outcomes were further analyzed.

\section{Methods}

\section{Patients}

The study was approved by the Committee on Research Ethics of Peking University First Hospital. Patients who were clinicopathologically diagnosed with D-ATIN from January 1, 2005, to December 31, 2018, and who were followed for at least 12 months were screened in a prospective cohort of ATIN patients in Peking University First Hospital as previously described [21]. The diagnosis of D-ATIN was made based on previously described criteria [22]. The presence of prominent interstitial inflammation in the nonfibrotic cortex and tubulitis was essential for the pathologic diagnosis of ATIN. All patients were screened for autoimmune diseases, malignancy and infectious diseases and accepted ophthalmological examinations to identify tubulointerstitial nephritis and uveitis syndrome (TINU) during their hospital stay. The cause of ATIN was reevaluated at every visit by the follow-up nephrologist group. Altogether, 81 patients with a final diagnosis of D-ATIN were enrolled in the current study.

\section{Clinical parameter evaluation and grouping for systemic inflammation}

Clinical parameters and laboratory data were documented. Acute kidney disease (AKD) was defined using the Kidney Disease: Improving Global Outcomes (KDIGO) criteria [23] and consensus report of the Acute Disease Quality Initiative (ADQI) 16 Workgroup [24]. The ESR was assessed by the Westergren method (Greiner bio-one, Germany), and CRP was detected by rate nephelometry (IMMAGE 800, Beckman Coulter, America) at the time of biopsy. The ESR and CRP levels were categorized by quartile and ranked from $0 \sim 3$ points (Table 1). The systemic inflammatory score (SIS) was calculated by combining the ESR and CRP points, and patients were classified into 3 systemic inflammation groups: low-SIS (score 0-1), medium-SIS (score 2-3) and high-SIS (score 4-6) groups. The scatter plot for CRP versus ESR values in different SIS groups was shown in Fig. 1.

\section{Evaluation of renal pathologic features}

All kidney biopsy tissues were processed for light microscopy, immunofluorescence, and electron microscopy. Semiquantitative scores for interstitial edema, infiltration, fibrosis, tubulitis and tubular atrophy were assessed by two pathologists referring to a modification of the Banff Working Classification [25, 26]. The activity index was the total of the scores for interstitial edema, infiltration and tubulitis. The chronicity index was the total of the scores for interstitial fibrosis and tubular atrophy.

\section{Immunofluorescence staining of infiltrated inflammatory cells} Infiltrating cells were identified by immunofluorescence staining with antibodies against CD3 (T lymphocytes, 1: 100, ZM-0417, ZSGB-BIO), CD20 (B lymphocytes, 1: 100, ZA-0293, ZSGB-BIO), CD38 (plasma cells, 1:250, ab108403, Abcam), CD68 (monocytes/macrophages, 1:

Table 1 Scoring system based on values of ESR and CRP

\begin{tabular}{lllll}
\hline CRP (mg/dL) & \multicolumn{5}{l}{ ESR (mm/hr) } \\
\cline { 2 - 5 } & $\begin{array}{l}<\mathbf{3 0} \\
\text { (0 Point) }\end{array}$ & $\begin{array}{l}\mathbf{3 0 - 5 0} \\
\text { (1 Points) }\end{array}$ & $\begin{array}{l}\mathbf{5 0 - 8 6} \\
\text { (2 Points) }\end{array}$ & (3 Points) \\
\hline$<3.6$ (0 Point) & 0 & 1 & 2 & 3 \\
$3.6-9.6$ (1 Points) & 1 & 2 & 3 & 4 \\
9.6-25.0 (2 Points) & 2 & 3 & 4 & 5 \\
$\geq 25.0$ (3 Points) & 3 & 4 & 5 & 6 \\
\hline
\end{tabular}




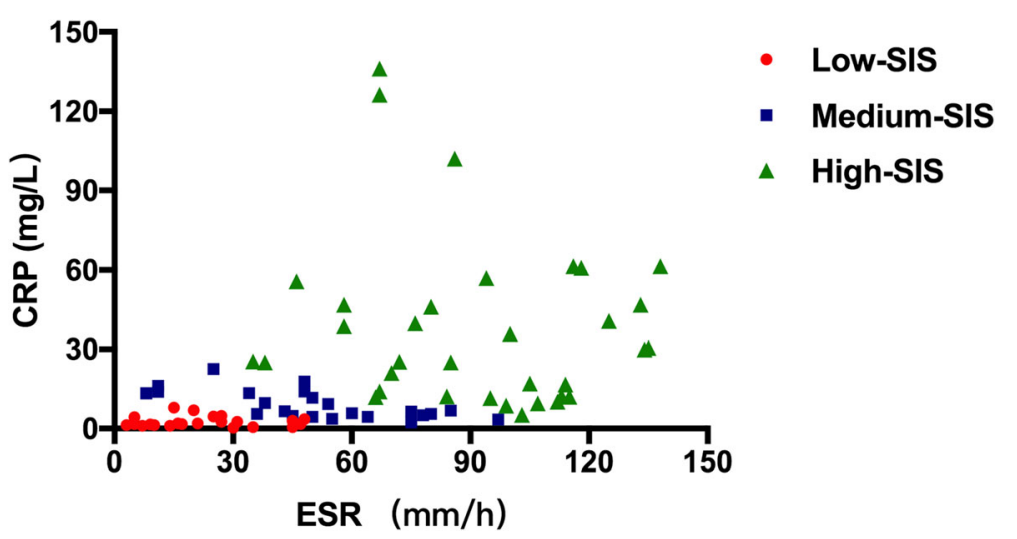

Fig. 1 Scatter plot for CRP versus ESR values in different SIS groups

500, ZM-0060, ZSGB-BIO), and neutrophil elastic protease antibodies (neutrophils, 1:200, GWB-8F72C4, Genway-Bio) and were counted at 400x magnification. Eosinophils were detected with hematoxylin and eosin staining and counted at 200x magnification. The quantitation of tubulointerstitial infiltration was determined by averaging the counts of five randomly selected fields. The mean values are expressed as cells per millimeter squared.

\section{Follow-up, renal recovery and renal outcome}

Serum creatine $(\mathrm{sCr})$ was routinely performed during the follow-up periods. Renal recovery was based on $\mathrm{sCr}$ levels at 6 months postbiopsy. Complete recovery was defined as improvement in $\mathrm{sCr}$ levels to within $25 \%$ of baseline (or to $133 \mu \mathrm{mol} / \mathrm{L}$ if the baseline was not available); partial recovery as a $>50 \%$ decrease in $\mathrm{sCr}$ level from its peak value but not reaching within $25 \%$ of its baseline value; and no recovery as the failure to meet the criteria for complete or partial recovery or remaining on renal replacement therapy (RRT). The renal outcome was defined by the estimated glomerular filtration rate (eGFR) at 12 months postbiopsy. The eGFR was calculated by the Chronic Kidney Disease Epidemiology Collaboration (CKD-EPI) equation [27] and expressed as milliliters per minute per $1.73 \mathrm{~m}^{2}$.

\section{Statistical analysis}

Analyses were performed using SPSS 22.0 Statistics software (IBM Corp., Armonk, NY) and GraphPad Prism version 6 (GraphPad Software, San Diego, CA). Categorical variables were expressed as counts and percentages. Continuous data with a normal distribution were presented as the mean and s.d., and those with an abnormal distribution were presented as the median and 25th75th percentile. To assess group differences, one-way analyses of variance and Chi-squared analyses were conducted. Bonferroni post hoc comparisons were computed when significant differences emerged. Spearman's rank correlation coefficient was used as a measure of correlations between the SIS and clinicopathological parameters. Multiple linear regression analysis was computed with the activity index as the dependent variable and with the following variables as explanatory variables: SIS, sex, age, disease course, and hemoglobin and eGFR levels at biopsy. Binary logistic regression analysis was used for predictors and ROC for cutoff points. A twosided $P<0.05$ was considered statistically significant.

\section{Results}

Baseline demographic and clinical data

As expressed in Table 2, the average age of the $81 \mathrm{D}$ ATIN patients was $45.4 \pm 12.9$ years, with a female predominance $(51 / 81,63.0 \%)$. The interval from the initiation of drug use to the diagnostic biopsy was 30 (14, $63)$ days. The majority of patients $(77 / 81,95.1 \%)$ were identified as having AKD, and 4 patients (4.9\%) were classified with CKD. Eighteen patients (22.2\%) required and initiated RRT before the biopsy. Seven patients (8.6\%) were oliguric. Twenty patients (24.7\%) had an allergic history. Common clinical features included digestive symptoms (61.7\%), weakness (48.1\%), fever (45.7\%) and rash (16.0\%). Beta-lactams, herbal medicine and nonsteroidal anti-inflammatory drugs were the most prevalent culprit agents $(45.7,39.5$ and $32.1 \%$, respectively). Thirty-eight patients (46.9\%) were identified as using more than one kind of culprit drug.

\section{Clinical relevance of the SIS in D-ATIN patients}

Of the $81 \mathrm{D}$-ATIN patients, the ESR was elevated in 70 (86.4\%), with an average level of $61.0 \mathrm{~mm} / \mathrm{hr}$. CRP was elevated in 44 patients $(54.3 \%)$, with a median value of $9.7 \mathrm{mg} / \mathrm{L}$ (Table 2). SISs evaluated by both ESR and CRP levels were positively correlated with $\mathrm{sCr}$ values at renal biopsy $(r=0.440 ; P<0.001)$, leukocyturia $(r=0.366 ; P=$ 
Table 2 Demographic and clinical features in different SIS groups

\begin{tabular}{|c|c|c|c|c|c|}
\hline Variables & $\begin{array}{l}\text { Total } \\
N=81\end{array}$ & $\begin{array}{l}\text { Low-SIS } \\
N=23\end{array}$ & $\begin{array}{l}\text { Medium-SIS } \\
N=24\end{array}$ & $\begin{array}{l}\text { High-SIS } \\
N=34\end{array}$ & $P$-value \\
\hline Female, n(\%) & $51(63.0)$ & $11(47.8)$ & $16(66.7)$ & $24(70.6)$ & 0.197 \\
\hline Age (year) & $45.4 \pm 12.9$ & $44.4 \pm 13.2$ & $47.5 \pm 13.1$ & $44.5 \pm 12.96$ & 0.643 \\
\hline Disease course (day) ${ }^{a}$ & $30(14,63)$ & $30(20,52)$ & $30(20,57)$ & $17(10,60)$ & 0.798 \\
\hline Allergic history, n(\%) & $20(24.7)$ & $6(26.1)$ & $6(25.0)$ & $8(23.5)$ & 0.975 \\
\hline Fever, n(\%) & $37(45.7)$ & $8(34.8)$ & $11(45.8)$ & $18(52.9)$ & 0.402 \\
\hline Rash, n(\%) & $13(16.0)$ & $4(17.4)$ & $3(12.5)$ & $6(17.6)$ & 0.847 \\
\hline $\mathrm{AKD}, \mathrm{n}(\%)$ & $77(95.1)$ & 19 (82.6) & $20(100.0)^{*}$ & $27(100.0)^{*}$ & 0.005 \\
\hline RRT, n(\%) & $18(22.2)$ & $1(4.3)$ & $9(37.5)^{*}$ & $8(23.5)^{*}$ & 0.023 \\
\hline Oliguria, n(\%) & $7(8.6)$ & $0(0)$ & $2(8.3)$ & $5(14.7)$ & 0.064 \\
\hline \multicolumn{6}{|l|}{ Suspected drug, n(\%) } \\
\hline Beta-lactams & $37(45.7)$ & $11(47.8)$ & $8(33.3)$ & $18(52.9)$ & 0.326 \\
\hline Herbal medicine & $32(39.5)$ & $13(56.5)$ & $10(41.7)$ & $9(26.5)$ & 0.072 \\
\hline NSAIDs & $26(32.1)$ & $7(30.4)$ & $7(29.2)$ & $12(35.3)$ & 0.868 \\
\hline PPIs & $6(7.4)$ & $2(8.7)$ & $4(16.7)$ & $0(0.0)^{\#}$ & 0.023 \\
\hline \multicolumn{6}{|l|}{ Laboratory tests } \\
\hline $\mathrm{SCr}$ at peak $(\mu \mathrm{mol} / \mathrm{L})$ & $322(220,531)$ & $269(158,329)$ & $417(242,646) *$ & $358(248,521) *$ & 0.021 \\
\hline $\mathrm{SCr}$ at biopsy $(\mu \mathrm{mol} / \mathrm{L})$ & $249(160,386)$ & $151(118,235)$ & $274(205,585) *$ & $321(185,449) *$ & $<0.001$ \\
\hline Hematuria, n (\%) & $21(25.9)$ & $3(13.0)$ & $9(37.5)$ & $9(26.5)$ & 0.160 \\
\hline Leukocyturia, n (\%) & $46(56.8)$ & $8(34.8)$ & $11(45.8)$ & $27(79.4)^{* *}$ & 0.002 \\
\hline UTP (g/24h) & $1.1(0.5,1.5)$ & $0.6(0.2,1.3)$ & $1.1(0.3,1.5)$ & $1.2(0.9,1.6) *$ & 0.038 \\
\hline U-NAG (U/L) & $26(15,47)$ & $37(16,57)$ & $20(13,33)$ & $25(15,48)$ & 0.215 \\
\hline U-a1MG (mg/L) & $171(74,238)$ & $76(26,218)$ & $154(73,252)$ & $206(136,271) *$ & 0.031 \\
\hline U-mAlb (mg/L) & $64(36,134)$ & $49(15,134)$ & $60(36,110)$ & $75(56,159)$ & 0.147 \\
\hline Renal glycosuria, n (\%) & $62(76.5)$ & $15(65.2)$ & $18(75.0)$ & $29(85.3)$ & 0.210 \\
\hline U-Osm decrease, n(\%) & $60(74.1)$ & $14(60.9)$ & $20(83.3)$ & $26(76.5)$ & 0.196 \\
\hline RTA, n (\%) & $43(53.1)$ & $6(26.1)$ & $15(62.5) *$ & $22(64.7) *$ & 0.009 \\
\hline Hemoglobin (g/L) & $104.0 \pm 16.6$ & $114.7 \pm 14.0$ & $103.0 \pm 20.2^{*}$ & $97.5 \pm 12.6^{*}$ & 0.001 \\
\hline Hypokalemia, n (\%) & $37(45.7)$ & $9(39.1)$ & $10(41.7)$ & $18(52.9)$ & 0.853 \\
\hline $\operatorname{ESR}(\mathrm{mm} / \mathrm{h})$ & $61.0 \pm 37.1$ & $25.6 \pm 14.3$ & $48.8 \pm 25.6^{*}$ & $91.5 \pm 28.5^{* \#}$ & $<0.001$ \\
\hline $\mathrm{CRP}(\mathrm{mg} / \mathrm{L})$ & $9.7(3.6,25.1)$ & $1.9(1.3,3.6)$ & $6.7(4.9,13.4) *$ & $27.6(12.3,49.0)^{* \#}$ & $<0.001$ \\
\hline $\lg G(g / L)$ & $15.6 \pm 4.4$ & $14.0 \pm 4.0$ & $15.2 \pm 3.3$ & $16.9 \pm 5.7$ & 0.681 \\
\hline C3 (mg/L) & $1.1 \pm 0.3$ & $0.9 \pm 0.2$ & $1.1 \pm 0.2^{*}$ & $1.3 \pm 0.2^{* \#}$ & $<0.001$ \\
\hline
\end{tabular}

Abbreviations: SIS systemic inflammatory score; $A K D$ acute kidney disease; RRT renal replacement therapy; NSAIDs non-steroidal anti-inflammatory drugs; PPIs proton pump inhibitors; $s C r$ serum creatinine; eGFR estimated glomerular filtration rate; UTP urinary total protein; U-KIM1 urinary kidney injury molecular 1; U-NAG urinary N-acetyl- $\beta$-D-glucosaminidase; U-a1MG urinary a1 microglobulin; U-mAlb urinary microalbumin; U-Osm urinary osmolality; RTA renal tubular acidosis; IgG immunoglobulin G; C3 complement 3

Normal range: U-NAG (0.3-12) U/L, U-a1MG (0-12) mg/L, U-mAlb (0-19) mg/L, ESR (0-15) mm/h, CRP (0-8) mg/L, lgG (7.2-16.9) g/L, C3 (0.6-1.5) mg/L

${ }^{a}$ Disease course was defined as the interval from initiation of drug use to the diagnostic biopsy

"compared with low-SIS group, $P<0.05 ;{ }^{*}$ compared with medium-SIS group, $P<0.05$

$0.001)$ and C3 levels $(r=0.533 ; P<0.001)$ (Additional Table 1).

Based on the SIS values, there were 23 patients in the low-SIS group, 24 in the medium-SIS group and 34 in the high-SIS group. There was no significant difference in age, sex, allergic manifestations, or causal medications among the three groups of patients. Patients in the low-SIS group had the mildest kidney injuries, with the lowest $\mathrm{sCr}$ levels at renal biopsy (median value: $151 \mu \mathrm{mol} / \mathrm{L}, P<0.001$ ), lowest RRT rate $(4.3 \%, P=0.023)$ and highest hemoglobin concentration $(114.7 \pm 14.0 \mathrm{~g} / \mathrm{L}, P=0.001)$. It is interesting to note that patients in the medium-SIS group tended to have higher peak sCr levels (median 417 vs $358 \mu \mathrm{mol} / \mathrm{L}$ ) and RRT rates (37.5\% vs $23.5 \%$ ) than those in the high-SIS group, yet their disease courses were relatively longer (median 30 vs 17 days) with lower levels of $\mathrm{sCr}$ at biopsy 
(median 274 vs $321 \mu \mathrm{mol} / \mathrm{L}$ ). In addition, patients in the high-SIS group had significantly higher C3 levels $(1.3 \pm 0.2$ vs $1.1 \pm 0.2 \mathrm{mg} / \mathrm{L}, P<0.001)$ with a greater prevalence of leukocyturia ( $79.4 \%$ vs $45.8 \%, P=0.002)$ than those in the medium-SIS group (Table 2).

\section{Pathological relevance of the SIS in D-ATIN patients}

Compared to patients in the low-SIS and medium-SIS groups, those in the high-SIS group had the highest degree of interstitial inflammation $(P<0.001)$ and the lowest degree of interstitial fibrosis $(P=0.030)$ (Table 3). The SIS was positively correlated with renal interstitial inflammatory cell infiltration $(r=0.508 ; P<0.001)$ and interstitial edema $(r=0.294 ; P=0.008)$ and inversely correlated with interstitial fibrosis $(r=-0.266 ; P=0.016) \quad$ (Additional Table 1). Multiple linear regression analysis demonstrated that only the SIS was significantly correlated with the renal activity index ( $\beta$ coefficient $=0.293, P=0.003$ ).

We next investigated renal interstitial inflammatory cell types through immunofluorescence staining. The number of each kind of interstitial inflammatory cell increased significantly with the increase in SISs (Table 3 ). When focusing on the constitution of inflammatory cells, the proportions of neutrophils $(7.5 \%$ vs $2.4 \%$ in medium-SIS vs $1.1 \%$ in low-SIS; $P<0.001$ ) and plasma cells $(12.6 \%$ vs $9.2 \%$ in medium-SIS vs $9.4 \%$ in low-SIS; $P=0.047$ ) were the highest in patients in the high-SIS group compared with those in the other two groups. There was no significant difference in the proportions of $\mathrm{T}$ lymphocytes, B lymphocytes or macrophages among the three groups of patients. Eosinophils, which favor a diagnosis of drug-induced ATIN, were also highest in the high-SIS group (median value: 3.8 vs 0.8 in mediumSIS vs 0.4 in low-SIS; $P<0.001$ ).

\section{Treatment and outcome among three groups with different SISs}

As shown in Table 4, prednisone was prescribed at a dosage of $30-40 \mathrm{mg} /$ day in all the patients. Additional immunosuppressive agents, such as mycophenolate, azathioprine and cyclophosphamide, were used in $27.2 \%$ $(22 / 81)$ of patients, with no significant difference among

Table 3 Pathology features in different SIS groups

\begin{tabular}{|c|c|c|c|c|c|}
\hline Variables & $\begin{array}{l}\text { Total } \\
N=81\end{array}$ & $\begin{array}{l}\text { Low-SIS } \\
N=23\end{array}$ & $\begin{array}{l}\text { Medium-SIS } \\
N=24\end{array}$ & $\begin{array}{l}\text { High-SIS } \\
N=34\end{array}$ & $P$-value \\
\hline \multicolumn{6}{|c|}{ Semiquantitative pathologic score } \\
\hline Activity index & $4(3,5)$ & $3(2,4)$ & $3(2,4)$ & $4(4,5)^{* \#}$ & $<0.001$ \\
\hline Interstitial edema & $1(0,1)$ & $1(0,1)$ & $1(0,1)$ & $1(1,1)^{*}$ & 0.044 \\
\hline Interstitial inflammation & $3(2,4)$ & $2(2,3)$ & $2(2,3)$ & $4(3,4)^{* \#}$ & $<0.001$ \\
\hline Tubulitis & $0(0,0)$ & $0(0,1)$ & $0(0,1)$ & $0(0,0)$ & 0.218 \\
\hline Chronicity index & $1(0,2)$ & $1(0,3)$ & $0(0,2)$ & $1(0,2)$ & 0.438 \\
\hline Interstitial fibrosis & $0(0,1)$ & $0(0,2)$ & $0(0,2)$ & $0(0,0)^{*}$ & 0.030 \\
\hline Tubular atrophy & $0(0,2)$ & $1(0,2)$ & $0(0,1)$ & $1(0,2)$ & 0.629 \\
\hline \multicolumn{6}{|c|}{ Interstitial inflammatory cell counts } \\
\hline Total cells ${ }^{a}$ & $391.5 \pm 135.5$ & $294.8 \pm 145.4$ & $378.2 \pm 97.8$ & $450.4 \pm 124.0^{*}$ & 0.001 \\
\hline T lymphocytes & $173.9 \pm 63.6$ & $137.6 \pm 72.3$ & $170.5 \pm 64.0$ & $195.5 \pm 50.08^{*}$ & 0.019 \\
\hline B lymphocytes & $45.4(30.4,62.0)$ & $34.7(14.4,41.6)$ & $45.6(30.7,60.4)$ & $56.4(40.1,69.1)^{*}$ & 0.040 \\
\hline Monocytes/macrophages & $101.8 \pm 34.8$ & $77.6 \pm 35.9$ & $90.7 \pm 30.5$ & $121.6 \pm 24.9^{* \#}$ & $<0.001$ \\
\hline Plasma cells & $43.8 \pm 24.3$ & $30.3 \pm 20.7$ & $32.6 \pm 19.3$ & $57.9 \pm 21.7^{* \#}$ & $<0.001$ \\
\hline Neutrophils & $15.4(4.9,33.0)$ & $2.9(1.7,13.8)$ & $7.9(4.7,20.4)$ & $31.7(15.7,48.8)^{* \#}$ & $<0.001$ \\
\hline Eosinophils $^{b}$ & $2.1(0.5,5.1)$ & $0.4(0.1,1.7)$ & $0.8(0.0,1.9)$ & $3.8(2.2,9.0) * \#$ & $<0.001$ \\
\hline \multicolumn{6}{|c|}{ Percentages of Interstitial inflammatory cells (\%) } \\
\hline T lymphocytes & $44.3 \pm 9.0$ & $46.9 \pm 8.5$ & $46.1 \pm 10.1$ & $41.7 \pm 8.2$ & 0.136 \\
\hline B lymphocytes & $11.7(9.1,14.2)$ & $10.6(8.8,14.5)$ & $12.6(9.4,14.9)$ & $11.5(8.7,14.0)$ & 0.263 \\
\hline Monocytes/macrophages & $26.0(21.3,29.9)$ & $25.8(21.5,30.9)$ & $25.7(19.9,30.1)$ & $26.2(23.4,29.1)$ & 0.670 \\
\hline Plasma cells & $10.9 \pm 5.2$ & $9.4 \pm 4.4$ & $9.2 \pm 5.7$ & $12.6 \pm 4.9^{* \#}$ & 0.047 \\
\hline Neutrophils & $4.2(1.6,7.8)$ & $1.1(0.9,5.4)$ & $2.4(1.7,4.8)$ & $7.5(4.3,11.7)^{* \#}$ & $<0.001$ \\
\hline
\end{tabular}

Abbreviations: SIS systemic inflammatory score

${ }^{a}$ Total cells count was the sum of T lymphocytes, B lymphocytes, macrophages, plasma cells and neutrophil under $400 \times$ magnification

${ }^{b}$ Eosinophils were counted under $200 \times$ magnification

*: compared with low-SIS group, $P<0.05$; ${ }^{\#}$ : compared with medium-SIS group, $P<0.05$ 
Table 4 Treatment and renal outcome in different SIS groups

\begin{tabular}{|c|c|c|c|c|c|}
\hline Variables & $\begin{array}{l}\text { Total } \\
N=81\end{array}$ & $\begin{array}{l}\text { Low-SIS } \\
N=23\end{array}$ & $\begin{array}{l}\text { Medium-SIS } \\
N=\mathbf{2 4}\end{array}$ & $\begin{array}{l}\text { High-SIS } \\
N=34\end{array}$ & $P$-value \\
\hline \multicolumn{6}{|l|}{ Immunosuppressive treatment, $\mathrm{n}(\%)$} \\
\hline Prednisone only & $52(64.2)$ & $19(82.6)$ & $15(62.5)$ & $18(52.9)$ & 0.071 \\
\hline Methylprednisolone pulse therapy & $18(22.2)$ & $0(0)$ & $6(25.0)^{*}$ & $12(35.3)^{*}$ & 0.007 \\
\hline Immunosuppressive medications & $22(27.2)$ & $4(17.4)$ & $8(33.3)$ & $10(29.4)$ & 0.436 \\
\hline \multicolumn{6}{|c|}{ Renal recovery at 6 months post-biopsy, n(\%) } \\
\hline Complete & $52(64.2)$ & $15(65.2)$ & $12(50.0)$ & $25(73.5)$ & 0.195 \\
\hline Partial & $28(34.6)$ & $7(30.4)$ & $12(50.0)$ & $9(26.5)$ & \\
\hline None & $1(1.2)$ & $1(4.3)$ & $0(0)$ & $0(0)$ & \\
\hline \multicolumn{6}{|c|}{ Renal outcome evaluated by eGFR (mL/min/1.73 m²) } \\
\hline$\geq 60$ & $47(58.0)$ & $16(69.6)$ & $8(33.3)^{*}$ & $18(67.6)^{\#}$ & 0.014 \\
\hline$<60$ & $28(42.0)$ & $7(30.4)$ & $16(66.7)$ & $11(32.4)$ & \\
\hline
\end{tabular}

Abbreviations: SIS systemic inflammatory score; eGFR estimated glomerular filtration rates; CKD chronic kidney disease; CRP C reactive protein; ESR erythrocyte sedimentation rate

*: compared with low-SIS group, $P<0.05$; : compared with medium-SIS group, $P<0.05$

the three groups $(P=0.436)$. Methylprednisolone pulse therapy was performed in $22.2 \%(18 / 81)$ of all patients, and none of the low-SIS patients received methylprednisolone pulse therapy.

Patients were followed for at least 12 months (range: 12-132 months, median 38 months). The high-SIS group tended to have more favorable renal restoration than the other two groups (Fig. 2). At 6 months postbiopsy, complete recovery was achieved in $73.5 \%$ of high-SIS patients, $50.0 \%$ of medium-SIS patients, and $65.2 \%$ of lowSIS patients $(P=0.195)$. A decreased eGFR $(<60 \mathrm{~mL} /$ $\min / 1.73 \mathrm{~m}^{2}$ ) was observed in $32.4 \%$ of high-SIS patients, $66.7 \%$ of medium-SIS patients, and $30.4 \%$ of low-SIS patients $(P=0.014)$ at 12 months postbiopsy (Table 4$)$. Adding SIS as a continuous variable to age and eGFR measured at biopsy made a small increase for the area under receiver operating characteristic curve by using the logistic regression analysis (from 0.696 to 0.731 for complete recovery at 6 months and from 0.852 to 0.875 for decreased eGFR at 12 months).

Fifty-four patients with severe renal dysfunction at the time of biopsy (eGFR $<30 \mathrm{~mL} / \mathrm{min} / 1.73 \mathrm{~m}^{2}$ ) were divided into high-score $(N=26)$ and low-score $(N=$ 28) subgroups based on the SIS. The eGFR values at biopsy were similar in the two subgroups $(14.3 \pm 7.8$ $\mathrm{mL} / \mathrm{min} / 1.73 \mathrm{~m}^{2}$ in high-score vs $17.7 \pm 8.5$ in lowscore, $P=0.131)$. At 12 months postbiopsy, the eGFR values were significantly higher in the high-score subgroup $\left(65.3 \pm 20.2\right.$ vs $52.9 \pm 20.9 \mathrm{~mL} / \mathrm{min} / 1.73 \mathrm{~m}^{2}$ in low-score, $P=0.032$ ).

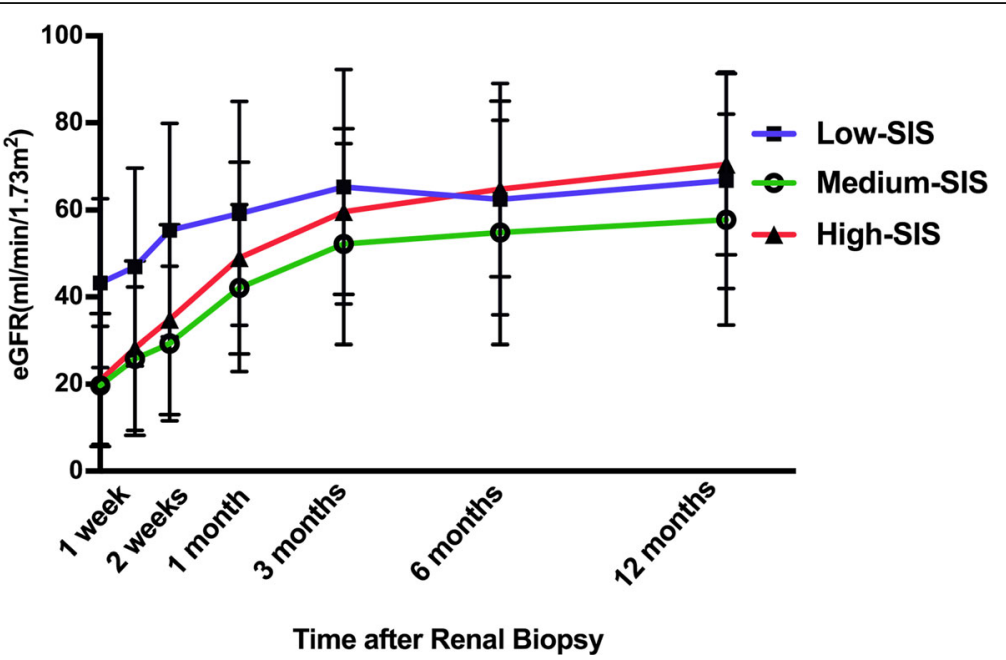

Fig. 2 The restoration of renal function in different SIS groups during the first year postrenal biopsy. Patients in both the medium-SIS and highSIS groups had significant renal dysfunction at the time of renal biopsy, and high-SIS patients presented with more rapid renal function restoration. Low-SIS patients had modest renal dysfunction and modest restoration of renal function 


\section{Discussion}

D-ATIN is a relatively common cause of AKI. Previously, the ESR and CRP were reported to be significantly elevated in D-ATIN patients [13, 28]. The current study first demonstrated that the SIS evaluated by the ESR and CRP was correlated with active renal tubulointerstitial inflammation and renal restoration in a prospective cohort of D-ATIN patients and therefore could help to aid therapeutic decisions when a renal biopsy is not acceptable or cannot be performed serially in this disease condition.

Patients with D-ATIN often present with a relatively insidious onset and a disease process of subacute renal dysfunction, with oliguria not commonly seen [13]. Therefore, a delayed diagnosis is likely to be encountered, especially when patients initially present at nonnephrology departments, as shown in our study, where the median time course from the initial use of suspicious drugs to diagnostic renal biopsy was 30 days, even exceeding 3 months in some cases. Once acute interstitial inflammation sets in, it can progress rapidly to a less reversible, more destructive fibrogenic process [29]. Therefore, the delay in diagnosis and treatment may result in complexity in the pathophysiologic process containing both active inflammation and fibrotic lesions in patients with D-ATIN. It is crucial to make a treatment decision with evidence that reflects renal interstitial active inflammation, yet renal biopsy, the gold standard for histological evaluation, is invasive and not acceptable for all patients. Following multivariate analysis, we observed that systemic inflammatory markers were closely correlated with renal inflammation regardless of the disease course. More importantly, it has been reported that DATIN patients might experience recurrent kidney injury during long-term follow-up due to various medications [21]. Elevated systemic inflammatory markers combined with abnormal urinary markers during follow-up may play an important role in reflecting the degree of renal inflammation and providing proper management for these patients.

During follow-up, for patients with similar eGFR values at biopsy, better renal outcomes were achieved in those with higher systemic inflammatory scores, suggesting that the SIS might serve as an indicator of renal outcome. Regarding pathological findings, the SIS was positively correlated with renal interstitial inflammatory cell infiltration, especially with neutrophils and eosinophils, which participate in the acute phase of inflammation [30], and was inversely correlated with interstitial fibrosis, which suggests its ability to reflect the activity of renal interstitial inflammatory injury in D-ATIN. A positive correlation was also observed between the SIS and plasma cell ratio. Plasma cell infiltration was found to be positively correlated with CRP levels and tubulointerstitial inflammation scores in antineutrophil cytoplasmic autoantibody-associated vasculitis [31]. The radical role of plasma cells in D-ATIN remains unclear, but we suppose that aggregated plasma cells in the kidney may play a role in local inflammation in the early phase. In summary, our findings indicate that the SIS may serve as a noninvasive biomarker for ongoing inflammatory processes and active kidney injury in DATIN patients, who might benefit from prompt treatment.

Our study has limitations related to the retrospective observational design. Due to the limited sample size of the patients that had both renal biopsy-proven D-ATIN and scheduled follow-up for at least 1 year, we were not able to test the scoring system in a new set of patients. Further study in a larger independent D-ATIN population is needed to validate our findings. CRP and ESR values are nonspecific markers that could be elevated in various conditions, such as infection and systemic autoimmune diseases. Therefore, the introduction of SISs in D-ATIN should be implemented after excluding these conditions.

\section{Conclusions}

Our study first demonstrated the relationship between systemic inflammation and local renal inflammation in D-ATIN. The SIS based on ESR and CRP values may serve as a marker of the activity of tubulointerstitial inflammation and assist with decision-making in the immunosuppressive treatment of D-ATIN.

\section{Supplementary Information}

Supplementary information accompanies this paper at https://doi.org/10. 1186/s12882-020-02175-z.

Additional file 1: Table S1. Correlation of systemic inflammatory

markers with clinicopathological parameters.

\section{Abbreviations}

D-ATIN: Drug-induced acute tubulointerstitial nephritis; CRP: C reactive protein; ESR: Erythrocyte sedimentation rate; SIS: Systemic inflammation score; HPF: High power field; sCr: Serum creatine; AKD: Acute kidney disease; KDIGO: Kidney Disease: Improving Global Outcomes; ADQI: The Acute Disease Quality Initiative; RRT: Renal replacement therapy; eGFR: Estimated glomerular filtration rate; CKD-EPI: Chronic Kidney Disease Epidemiology Collaboration; CKD: Chronic kidney disease; NSAIDs: Non-steroidal antiinflammatory drugs; PPIs: Proton pump inhibitors; UTP: Urinary total protein; U-KIM1: Urinary kidney injury molecular 1; U-NAG: Urinary N-acetyl- $\beta-D-g l u-$ cosaminidase; U-a1MG: Urinary a1 microglobulin; U-mAlb: Urinary microalbumin; U-Osm: Urinary osmolality; RTA: Renal tubular acidosis; IgG: Immunoglobulin G; C3: Complement 3

\section{Acknowledgments}

The results presented in this paper have not been published previously in whole or part, except in abstract form at 3rd Asia Pacific AKI CRRT 2019 Congress. We have obtained the necessary permission from the copyright holder. We thank the patient for devotion. 


\section{Authors' contributions}

Research idea and study design: TS, XJZ, GL and LY. Reacher Data acquisition: XZZ, YHG, DMX, JWH and PPS. Histological examination: SXW JWH, PPS and YJ. Data analysis/interpretation and statistical analysis: XZZ YHG and DMX. Draft: XZZ. Revise: LY. All authors read and approved the final manuscript.

\section{Funding}

This study was supported by grants from the National Natural Science Foundation of China (No. 91742205 and No. 81625004) and the National Science and Technology Major Projects for "Major New Drugs Innovation and Development" of China (No. 2017ZX09304028) and Peking University Clinical Scientist Program (BMU2019LCKXJ002). The funder played a role in the design of the study, follow-up of the patients, interpretation of the data and writing of the manuscript.

\section{Availability of data and materials}

The datasets used and analyzed during the current study are available from the corresponding author upon reasonable request.

\section{Ethics approval and consent to participate}

The study was performed in compliance with the Declaration of Helsinki and approved by the Ethics Committee of Peking University First Hospital (approval number 2017[1280]). Written informed consent for obtaining tissue, blood and urine samples was obtained from each participant or their parents or guardian (for participants under 16 years old).

\section{Consent for publication}

Not applicable.

\section{Competing interests}

The authors declare that they have no competing interests.

\section{Author details}

'Renal Division, Peking University First Hospital, Peking University Institute of Nephrology; Key Laboratory of Renal Disease, Ministry of Health of China; Key Laboratory of Chronic Kidney Disease Prevention and Treatment (Peking University), Ministry of Education, Beijing 100034, People's Republic of China. ${ }^{2}$ Renal Pathology Center, Peking University Institute of Nephrology, Beijing 100034, People's Republic of China. ${ }^{3}$ Cardiovascular Division, Fourth Clinical Medical College, Guangzhou University of Chinese Medicine, Shenzhen 518033, People's Republic of China. ${ }^{4}$ Laboratory of Electron Microscopy, Pathological Center, Peking University First Hospital, Beijing 100034, People's Republic of China.

Received: 10 December 2019 Accepted: 17 November 2020 Published online: 26 November 2020

\section{References}

1. Raghavan R, Eknoyan G. Acute interstitial nephritis - a reappraisal and update. Clin Nephrol. 2014;82(3):149-62.

2. Praga $M$, Sevillano A, Aunon $P$, et al. Changes in the aetiology, clinical presentation and management of acute interstitial nephritis, an increasingly common cause of acute kidney injury. Nephrol Dial Transplant. 2015;30(9): 1472-9.

3. Clarkson MR, Giblin L, O'Connell FP, et al. Acute interstitial nephritis: clinical features and response to corticosteroid therapy. Nephrol Dial Transplant. 2004;19(11):2778-83.

4. Michel DM, Kelly CJ. Acute interstitial nephritis. J Am Soc Nephrol. 1998;9(3): 506-15.

5. Perazella MA, Markowitz GS. Drug-induced acute interstitial nephritis. Nat Rev Nephrol. 2010;6(8):461-70.

6. Schwarz A, Krause PH, Kunzendorf $U$, et al. The outcome of acute interstitial nephritis: risk factors for the transition from acute to chronic interstitial nephritis. Clin Nephrol. 2000;54(3):179-90.

7. Muriithi AK, Leung N, Valeri AM, et al. Biopsy-proven acute interstitial nephritis, 1993-2011: a case series. Am J Kidney Dis. 2014;64(4):558-66.

8. Muriithi AK, Leung N, Valeri AM, et al. Clinical characteristics, causes and outcomes of acute interstitial nephritis in the elderly. Kidney Int. 2015:87(2): 458-64.
9. Perazella MA. Diagnosing drug-induced AIN in the hospitalized patient: a challenge for the clinician. Clin Nephrol. 2014;81(6):381-8.

10. Valluri A, Hetherington $L$, McQuarrie $E$, et al. Acute tubulointerstitial nephritis in Scotland. QJM. 2015:108(7):527-32.

11. Krishnan N, Perazella MA. Drug-induced acute interstitial nephritis: pathology, pathogenesis, and treatment. Iran J Kidney Dis. 2015;9(1):3-13.

12. Ulinski T, Sellier-Leclerc AL, Tudorache $E$, et al. Acute tubulointerstitial nephritis. Pediatr Nephrol. 2012;27(7):1051-7.

13. Li C, Su T, Chu R, et al. Tubulointerstitial nephritis with uveitis in Chinese adults. Clin J Am Soc Nephrol. 2014;9(1):21-8.

14. Vila LM, Alarcon GS, McGwin G Jr, et al. Systemic lupus erythematosus in a multiethnic cohort (LUMINA): XXIX. Elevation of erythrocyte sedimentation rate is associated with disease activity and damage accrual. J Rheumatol. 2005;32(11):2150-5.

15. Eudy AM, Vines Al, Dooley MA, et al. Elevated C-reactive protein and selfreported disease activity in systemic lupus erythematosus. Lupus. 2014; 23(14):1460-7.

16. Yang M, Ma N, Fu H, et al. Hematocrit level could reflect inflammatory response and disease activity in patients with systemic lupus Erythematosus. Clin Lab. 2015;61(7):801-7.

17. Dima A, Opris D, Jurcut $C$, et al. Is there still a place for erythrocyte sedimentation rate and C-reactive protein in systemic lupus erythematosus? Lupus. 2016;25(11):1173-9.

18. Nielung L, Christensen R, Danneskiold-Samsoe B, et al. Validity and agreement between the 28-joint disease activity score based on C-reactive protein and erythrocyte sedimentation rate in patients with rheumatoid arthritis. Arthritis. 2015;2015:401690

19. Martire MV, Marino Claverie L, Duarte V, et al. Factors associated with sustained remission in patients with rheumatoid arthritis. Reumatol Clin. 2015;11(4):237-41.

20. Monach PA, Warner RL, Tomasson G, et al. Serum proteins reflecting inflammation, injury and repair as biomarkers of disease activity in ANCAassociated vasculitis. Ann Rheum Dis. 2013;72(8):1342-50.

21. Su T, Gu Y, Sun P, et al. Etiology and renal outcomes of acute tubulointerstitial nephritis: a single-center prospective cohort study in China Nephrol Dial Transplant. 2018;33(7):1180-8.

22. Wu Y, Yang $L$, Su T, et al. Pathological significance of a panel of urinary biomarkers in patients with drug-induced tubulointerstitial nephritis. Clin J Am Soc Nephrol. 2010;5(11):1954-9.

23. Kidney Disease. Improving Global Outcomes (KDIGO) Acute Kidney Injury Work Group: KDIGO clinical practice guideline for acute kidney injury. Kidney Int. 2012;2:138.

24. Chawla LS, Bellomo R, Bihorac A, et al. Acute kidney disease and renal recovery: consensus report of the acute disease quality initiative (ADQI) 16 workgroup. Nat Rev Nephrol. 2017;13(4):241-57.

25. Solez K, Colvin RB, Racusen LC, et al. Banff 07 classification of renal allograft pathology: updates and future directions. Am J Transplant. 2008;8(4):753-60.

26. Racusen LC, Solez K, Colvin RB, et al. The Banff 97 working classification of renal allograft pathology. Kidney Int. 1999;55(2):713-23.

27. Levey AS, Stevens LA, Schmid CH, et al. A new equation to estimate glomerular filtration rate. Ann Intern Med. 2009;150(9):604-12.

28. Simpson IJ, Marshall MR, Pilmore $H$, et al. Proton pump inhibitors and acute interstitial nephritis: report and analysis of 15 cases. Nephrology (Carlton). 2006;11(5):381-5.

29. Gonzalez E, Gutierrez E, Galeano C, et al. Early steroid treatment improves the recovery of renal function in patients with drug-induced acute interstitial nephritis. Kidney Int. 2008;73(8):940-6.

30. Kolaczkowska E, Kubes P. Neutrophil recruitment and function in health and inflammation. Nat Rev Immunol. 2013;13(3):159-75.

31. Masuzawa N, Nishimura A, Mihara Y, et al. Clinicopathological analysis of ANCA-associated glomerulonephritis focusing on plasma cell infiltrate. Clin Exp Nephrol. 2019;23(12):1373-81.

\section{Publisher's Note}

Springer Nature remains neutral with regard to jurisdictional claims in published maps and institutional affiliations. 